Elk1 $1^{+/ 0}$ controls $(9.40,2.24$ median relative expression, respectively $n=3$ ). We therefore performed immunohistochemical staining for $\alpha \mathrm{SMA}$ in the lungs of mice aged to 1 year and demonstrated visible increases in expression of $\alpha \mathrm{SMA}$ in the alveolar epithelium of $E l k 1^{-/ 0}$ mice but not in $E l k 1^{+/ 0}$ controls.

Conclusion These data suggest that Elk1 gene deletion result in age-related early fibrotic changes associated with the development of pulmonary fibrosis.

\section{S119 MAPPING MOUSE MODELS OF SEVERE ASTHMA ONTO HUMAN DISEASE}

${ }^{1} \mathrm{~K}$ Kazi, ${ }^{2} \mathrm{IM}$ Adcock, ${ }^{3} \mathrm{~S}$ Pavlidis. ${ }^{1}$ Imperial College London, London, UK; ${ }^{2}$ National Heart and Lung Institute, London, UK; ${ }^{3}$ Data Science Institute, London, UK

\subsection{6/thoraxjnl-2017-210983.125}

Introduction Severe asthma represents a significant unmet need in terms of therapeutics. Drug development in asthma has been slow and expensive and one of the reasons for this is that positive findings for drugs tested in preclinical models have not readily translated into successful therapies in man.

Aims and Objectives We sought to improve the predictive power of existing models of asthma by using novel bioinformatics techniques to align these models with subsets of human asthma.

Methods We applied differential gene expression analysis to transcriptomic data from whole lung samples of 6 murine models of asthma and oxidative stress to produce gene signatures that represented each model. These signatures were then used to calculate enrichment scores (ESs) for transcriptomic data from bronchial biopsies taken from 81 asthmatic and 26 healthy subjects from the U -BIOPRED cohort using gene set variation analysis. These ESs were taken as an index of similarity between each model and each patient and were used to drive further analyses using topological data analysis and goodness of fit modelling.

Results We found that no single mouse model was aligned well with all asthmatics. We identified three clusters of patients who were represented to varying degrees by different mouse models and who displayed clinical features that aligned well with phenotypes of asthmatics identified previously by clustering analyses based upon clinical features and biological markers. Patients in cluster $\chi$ were defined by neutrophilic sputum, later onset of disease, higher incidence of sinusitis, more frequent exacerbations and more airflow limitation. Patients in cluster Y1 showed significantly lower sputum neutrophils, a trend towards higher sputum eosinophils, a significantly later onset of airways disease and a trend towards higher BMI. Patients in cluster Y2 showed a significantly higher percentage of neutrophils in the blood, a trend towards increased sputum lymphocytes and were more likely to identify aspirin as a trigger $(\mathrm{p}=0.06)$.

Conclusion Our evidence supports the assertion that it is possible, on a transcriptional level, to align mouse models of asthma to subsets of human asthma and that doing so may have significant implications for the expedience of drug development in asthma.

\section{S120 GENE THERAPY FOR PULMONARY ALVEOLAR PROTEINOSIS}

${ }^{1} \mathrm{~N}$ Atsumi, ${ }^{1} \mathrm{~A}$ Pilou, ${ }^{2}$ Pringle, ${ }^{2} \mathrm{RC}$ Ashworth, ${ }^{3} \mathrm{C}$ Meng, ${ }^{3} \mathrm{M}$ Chan, ${ }^{2} \mathrm{DR}$ Gill, ${ }^{2} \mathrm{~S}$ Hyde, ${ }^{4} \mathrm{C}$ Morgan, ${ }^{1} \mathrm{EWFW}$ Alton, ${ }^{3} \mathrm{U}$ Griesenbach. ${ }^{1}$ Department of Gene Therapy, NHLI, Imperial College London and UK Gene Therapy Consortium, London, UK; ${ }^{2}$ Radcliffe Department of Medicine, Oxford University and Gene Therapy Consortium, London, UK; ${ }^{3}$ Department of Gene Therapy, NHLI, Imperial College London and Raddliffe Department of Medicine, Oxford University, London, UK; ${ }^{4}$ Royal Brompton Hospital, London, UK

\subsection{6/thoraxjnl-2017-210983.126}

Introduction Pulmonary alveolar proteinosis (PAP) is characterised by accumulation of surfactant in the terminal airways. Granulocyte-Macrophage Colony-Stimulating-Factor (GM-CSF) stimulates alveolar macrophages to clear surfactant. The presence of GM-CSF autoantibodies in autoimmune PAP (aPAP) leads to surfactant build-up and impaired gas exchange. This causes respiratory symptoms and can ultimately be fatal due to hypoxaemic respiratory failure. We hypothesise that lentivirus-mediated gene transfer of GM-CSF may be suitable to treat aPAP and propose to assess efficacy of GM-CSF gene transfer in GM-CSF knockout mice, which recapitulate aPAP lung disease. The murine GM-CSF (mGM-CSF) cDNA was cloned into a lentiviral vector, which was pseudotyped with the $\mathrm{F}$ and $\mathrm{HN}$ proteins from Sendai virus to enable efficient lung transduction (rSIV.F/HN-mGM-CSF).

Methods and Results To confirm if the vector produces mGMCSF we first transduced A549 cells with multiplicity of infection (MOI) of $0.1-100$ ( $n=6 /$ group). 48 hours after transduction dose-related mGM-CSF expression was confirmed in the medium. We next assessed whether the mGM-CSF produced after gene transfer was biologically active by comparing the proliferation rate of FDC-P1 cells, a mGM-CSF-dependent mouse myeloid progenitor cell line, in the presence of gene therapy- produced mGM-CSF $(0.001-10 \mathrm{ng} / \mathrm{ml})$ and purchased recombinant $\mathrm{mGM}-\mathrm{CSF}$ protein $(\mathrm{n}=6 /$ group). The dose-related proliferation rates in both conditions were similar (figure 1ss). In preliminary experiments, we next assessed whether gene transfer led to GM-CSF production in vivo. rSIV.F/HN-mGMCSF (1e7 transduction units (TU)/mouse) was administered to wild-type mice by nasal "sniffing". Control mice remained untransduced ( $n=3 /$ group). mGM-CSF levels were quantified in lung tissue homogenate and broncho-alveolar lavage fluid (BALF) 14 days after gene transfer. mGM-CSF levels in untreated mice were below the limit of detection of the ELISA, but high levels of mGM-CFS were detectable in lung tissue (median 825 (range 460-3790) pg/mg) and BALF (median: 3330 (range 2307-7958) pg/ml).

Conclusion rSIV.F/HN-mGM-CSF produced mGM-CSF in vitro and in vivo. The biological function of the protein was confirmed in vitro and evaluation of mGM-CSF gene transfer efficacy in murine aPAP model is ongoing. 